\title{
Fiberwise localization and the cube theorem
}

\author{
David Chataur and Jérôme Scherer*
}

\begin{abstract}
In this paper we explain when it is possible to construct fiberwise localizations in model categories. For pointed spaces, the general idea is to decompose the total space of a fibration as a diagram over the category of simplices of the base and replace it by the localized diagram. This of course is not possible in an arbitrary category. We have thus to adapt another construction which heavily depends on Mather's cube theorem. Working with model categories in which the cube theorem holds, we propose a few equivalent conditions under which fiberwise nullifications exist. We show that these techniques apply to yield a fiberwise plus-construction for differential graded algebras over cofibrant operads.
\end{abstract}

Mathematics Subject Classification (2000). Primary 55P60, 55U35, 18D50; Secondary 18G55, 55P48.

Keywords. Localization, fiberwise, plus-construction, model category, operads, cube theorem.

\section{Introduction}

Mather's cube theorem states that the top face of a cube of spaces whose bottom face is a homotopy push-out and all vertical faces are homotopy pull-backs is again a homotopy push-out ([13, Theorem 25]). This theorem is one of the very few occurrences of a situation where homotopy limits and colimits commute. It is actually related to a theorem of Puppe about commuting fibers and push-outs ([16]), and also to Quillen's Theorem B in [18]. Doeraene's work on $J$-categories has incorporated the cube theorem as an axiom in pointed model categories and allowed him to study the L.S.-category in an abstract setting ([6]). Roughly speaking a $J$-category is a model category in which the cube theorem holds. Such a model category is very suitable for studying the relationship between a localization functor (constructed by means of certain homotopy colimits) and fibrations.

*The first author is supported by the Marie Curie Grant HPMF-CT-2001-01179. The second author is supported by the program Ramón y Cajal, MEC, Spain, as well as by MEC grant MTM2004-06686. 
Recall that a localization functor in a model category $\mathcal{M}$ is any coaugmented homotopically idempotent functor $L: \mathcal{M} \rightarrow \mathcal{M}$, the coaugmentation being a natural transformation $\eta$ : Id $\rightarrow L$. The localization functors $L_{f}$, which "invert" the morphism $f$, have been constructed for spaces by Dror Farjoun and exist in rather general model categories by work of Hirschhorn. We are looking for an existence theorem of fiberwise localization, i.e. a construction which associates to a fibration $F \rightarrow E \rightarrow B$ another fibration together with a natural transformation

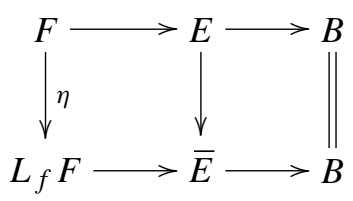

where $E \rightarrow \bar{E}$ is an $L_{f}$-equivalence. For pointed spaces, the most elegant construction of fiberwise localization is due to Dror Farjoun (in [8, Theorem 1.F.3]). His idea is to decompose the total space of a fibration as a diagram over the category of simplices of the base and replace it by the corresponding localized diagram. In certain particular settings, some authors used other constructions (May [15], Dwyer, Miller, and Neisendorfer in [9] for completions, Casacuberta and Descheemaker in [3] in the category of groups), but none of these can be adapted in model categories. Farjoun's construction in particular is useless in any category where the homotopy colimit of a diagram with contractible values is trivial (such as differential graded algebras over an operad, see the applications below). We prove the following:

Theorem 4.3. Let $\mathcal{M}$ be a model category which is pointed, left proper, cellular and in which the cube axiom holds. Assume also that $\mathcal{M}$ either has a set of detectors, or satisfies the ladder axiom. Let $L_{f}: \mathcal{M} \rightarrow \mathcal{M}$ be a localization functor, $F \rightarrow E \rightarrow B$ be a fibration, and assume that $L_{f}(\Omega B \times X) \simeq L_{f} \Omega B \times L_{f} X$ for any $X \in \mathcal{M}$. Then there exists a fiberwise localization, i.e. a natural transformation to a new fibration

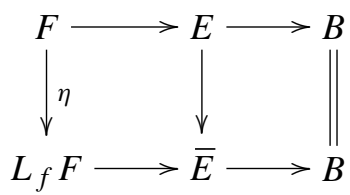

where $E \rightarrow \bar{E}$ is an $f$-local equivalence.

Here detectors are some special objects playing the role of spheres. They exist in the category of pointed spaces, algebras over an operad, $G$-equivariant spaces, spectra, etc. So basically fiberwise localizations exist when the localization of a product is weakly equivalent to the product of the localizations, in other words when 
trivial fibrations admit a fiberwise localization! Because it is so difficult to compute the localization of a product, we will mostly deal with nullification functors. They are localization functors $L_{f}$ where the map $f$ is of the form $A \rightarrow *$ and are written $P_{A}$. Recall that the image of $P_{A}$ consists in all objects $X$ for which the space map $\left(A, P_{A} X\right)$ of (pointed) maps is contractible. In this context we will be able to replace the condition on the product by one which can be actually checked in specific examples and is stated in terms of the join.

The join axiom. The join axiom is satisfied for an object $A$ if the join $X * A$ is killed by $P_{A}$ for any object $X$, i.e. $P_{A}(X * A) \simeq *$.

One should not however expect fiberwise nullification to exist without further restriction, as shown by Hirschhorn's Example 4.2 of pointed spaces. Requiring that the base of the fibration be path connected, we show in Theorem 5.3 that fiberwise nullifications exist in pointed model categories satisfying the cube and the join axioms.

For such categories the join axiom is actually necessary and we characterize the model categories for which fiberwise nullifications exist, see Theorem 5.5.

We work in the last part of the paper with the category of differential graded algebras over a cofibrant operad. As shown in [1] this is a model category and we prove that it satisfies the cube axiom. Therefore the plus-construction developed in [5] has a fiberwise analogue. Let us only say that the plus-construction performed on a (non-negatively graded) $\mathcal{O}$-algebra $B$ kills the maximal $\mathcal{O}$-perfect ideal in $\pi_{0} B$ and preserves Quillen homology. As a direct consequence we get the following result which is classical for spaces.

Theorem 6.7. Let $\mathcal{O}$-alg be the category of non-negatively graded differential graded algebras over a cofibrant operad $\mathcal{O}$. For any $\mathcal{O}$-algebra $B$, denote by $B \rightarrow B^{+}$the plus construction. The homotopy fiber $A B=\operatorname{Fib}\left(B \rightarrow B^{+}\right)$is then acyclic with respect to Quillen homology.

Acknowledgements. We would like to thank Jean-Paul Doeraene, Gustavo Granja, and Mark Hovey for helpful comments. We also thank the referee for his careful reading and for pointing out two errors in a previous version.

\section{Notation and terminology}

We recall here the basics on model categories and localization. The main reference is Hirschhorn's book [11], but there are of course classical references such as [17] on model categories, [2] and [8] on localization.

In a model category $\mathcal{M}$ which is pointed, the terminal object coincides with the initial one and is denoted by $*$. In such a category the homotopy fiber $\operatorname{Fib}(p)$ of a 
map $p: E \rightarrow B$ is defined as the homotopy pull-back of the diagram $* \rightarrow B \leftarrow E$. If $\mathcal{M}$ is not pointed, there may exist many morphisms $* \rightarrow B$ and thus distinct fibers.

We will assume the category is left proper, meaning that the push-out of a weak equivalence along a cofibration is again a weak equivalence. Finally we require $\mathcal{M}$ to be cellular as defined in [11, Definition 12.1.1]. Basically the small object argument applies in a cellular model category, as one has a set $I$ of generating cofibrations $A_{i} \rightarrow B_{i}$ which replace the usual spheres. Every object has a cofibrant replacement by a cell complex by [11, Theorem 11.2.7] and for every cofibrant object there exists a cardinal $\kappa$ such that any morphism from it to a telescope of length $\lambda \geq \kappa$ made of cofibrations factorizes through an object of this telescope (smallness of cofibrant objects, see [11, Theorem 12.4.3]). By a telescope of length $\lambda$ (or $\lambda$-sequence, [11, Definition 10.2.1]) we mean a diagram of the form $X_{0} \rightarrow X_{1} \rightarrow \cdots \rightarrow X_{\beta} \rightarrow \cdots$ for $\beta<\lambda$ such that each morphism is a cofibration and for every limit ordinal $\gamma<\lambda$ the map colim ${ }_{\alpha<\gamma} X_{\alpha} \rightarrow X_{\gamma}$ is an isomorphism.

Recall that $\aleph_{0}$ is the countable cardinal. An object $G$ in $\mathcal{M}$ is said to be finite (or $\aleph_{0}$-small) if any morphism from $G$ into a (countable or possibly transfinite) telescope factors through some object of the telescope. Thus $\left[G, \operatorname{hocolim}\left(A_{0} \rightarrow\right.\right.$ $\left.A_{1} \rightarrow \cdots \rightarrow A_{\beta} \rightarrow \cdots\right] \cong \operatorname{colim}\left(\left[G, A_{0}\right] \rightarrow\left[G, A_{1}\right] \rightarrow \cdots \rightarrow\left[G, A_{\beta}\right] \rightarrow \cdots\right)$.

In general weak equivalences are detected by mapping spaces out of the generating cofibrations (this can be deduced from [11, Theorem 17.7.7]). In order to have a more handy criterion we will assume sometimes that our model category contains some special objects playing the role of spheres.

Definition 1.1. A set of finite objects $g$ is called a set of detectors if they detect weak equivalences, i.e. a morphism $X \rightarrow Y$ is a weak equivalence if and only if it induces a bijection $[G, X] \cong[G, Y]$ for any $G \in G$.

This condition is satisfied in all the examples we have in mind: Spaces, spectra, algebras over an operad, etc. In [12, Corollary 7.4.4] Hovey shows that any cofibrantly generated pointed model category has a set of weak generators, which detect contractible objects, namely the cofibers of the generating cofibrations. In general these weak generators are not detectors, as is illustrated by the case of pointed spaces (the cofibers are all connected). The key point where detectors are used in this paper is to get a property about telescopes (cf. Lemma 2.6).

Let $\mathcal{M}$ be a cellular model category and $f: A \rightarrow B$ be any morphism in $\mathcal{M}$. Recall that it is always possible to construct mapping spaces up to homotopy in $\mathcal{M}$ even though we do not assume $\mathcal{M}$ to be a simplicial model category (see [12, Section 5.4] or [11, Chapter 17]). Thus we can define an object $Z \in \mathcal{M}$ to be $f$-local if $f$ induces a weak equivalence $f^{*}: \operatorname{map}(B, Z) \simeq \operatorname{map}(A, Z)$. A map $g: X \rightarrow Y$ is an $f$-local equivalence if it induces a weak equivalence on mapping spaces $g^{*}: \operatorname{map}(Y, Z) \rightarrow \operatorname{map}(X, Z)$ for any $f$-local object $Z$. Hirschhorn shows 
in [11, Theorem 4.1.1] that there exists a coaugmented functor $L_{f}: \mathcal{M} \rightarrow \mathcal{M}$ such that the coaugmentation $\eta: X \rightarrow L_{f} X$ is an $f$-local equivalence to an $f$-local object. This functor is called $f$-localization.

When working with a nullification functor $P_{A}$ for some object $A \in \mathcal{M}$, we say that $X$ is $A$-acyclic or killed by $A$ if $P_{A} X \simeq *$. By universality this is equivalent to map $(X, Z) \simeq *$ for any $A$-local object $Z$, or even better to the fact that any morphism $X \rightarrow Z$ to an $A$-local object is homotopically trivial.

\section{The cube axiom}

In general we do not know if it is possible to localize fiberwise in any (pointed, left proper, cellular) model category. We will thus work in model categories satisfying an extra condition.

Definition 2.1. A model category $\mathcal{M}$ satisfies the cube axiom if for every commutative cubical diagram in $\mathcal{M}$ in which the bottom face is a homotopy push-out square and all vertical faces are homotopy pull-back squares, then the top face is a homotopy push-out square as well.

Mather proved the cube theorem for spaces in [13, Theorem 25] and Doeraene introduced it as an axiom for model categories. His paper [6] contains a very useful appendix with several examples of model categories satisfying this rather strong axiom.

Example 2.2. Any stable model category satisfies the cube axiom. Indeed homotopy push-outs coincide with homotopy pull-backs, so that this axiom is a tautology. On the other hand the category of groups does not satisfy the cube axiom. Let us give an easy counter-example by considering the push-out of $(\mathbb{Z} \leftarrow * \rightarrow \mathbb{Z})$, which is a free group on two generators $a$ and $b$. The pull-back along the inclusion $\mathbb{Z}\langle a b\rangle \hookrightarrow \mathbb{Z}\langle a\rangle * \mathbb{Z}\langle b\rangle$ is obviously not a push-out diagram. However fiberwise localizations exist in the category of groups as shown by the recent work of Casacuberta and Descheemaker [3].

The following proposition claims that under very special circumstances the homotopy push-out of the homotopy fibers coincides with the homotopy fiber of the homotopy push-outs. In the category of spaces this is originally due to Puppe, see [16]. The close link between the cube theorem and Puppe's theorem was already well known to Mather and Walker, as can be seen in [14]. 
Proposition 2.3. Let $\mathcal{M}$ be a pointed model category in which the cube axiom holds. Consider natural transformations between push-out diagrams:

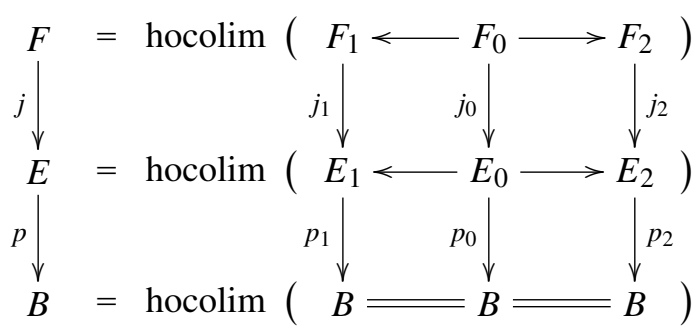

Assume that $F_{i}=\operatorname{Fib}\left(p_{i}\right)$ for any $0 \leq i \leq 2$. Then $F$ is the homotopy fiber $\operatorname{Fib}(p)$.

Proof. Denote by $k: G \rightarrow E$ the homotopy fiber of $p$. We show that $G$ and $F$ are weakly equivalent. Let us construct a cube by pulling-back $E_{i} \rightarrow E$ along $k$. The bottom face consists thus in the middle row of the above diagram and the top face consists in the homotopy pull-backs of $E_{i} \rightarrow E \leftarrow G$, which are the same as the homotopy pull-backs of $E_{i} \rightarrow B \leftarrow *$, i.e. $F_{i}$. The cube axiom now states that the top face is a homotopy push-out and we are done.

This result will be the main tool in constructing fiberwise localization in $\mathcal{M}$. In his paper [6] on L.S.-category, Doeraene used the cube axiom in a very similar fashion to study fiberwise joins. Indeed Ganea's characterization of the L.S.-category uses iterated fibers of push-outs over a fixed base space. The same ideas have also been used in [7]. The next lemmas state commutation rules of homotopy colimits with products. Notice that we do not claim that products commute with coproducts.

Lemma 2.4. Let $\mathcal{M}$ be a model category in which the cube axiom holds. Let $D$ be the homotopy push-out in $\mathcal{M}$ of the diagram $A \leftarrow B \rightarrow C$. Then, for any object $X \in \mathcal{M}$, $X \times D$ is the homotopy push-out of the diagram $X \times A \leftarrow X \times B \rightarrow X \times C$.

Proof. It suffices to consider the cube obtained by pulling back the mentioned pushout square along the canonical projection $X \times D \rightarrow D$.

The following axiom is a very natural analogue of the cube axiom. It says that telescopes commute with homotopy pull-backs.

Definition 2.5. A model category $\mathcal{M}$ satisfies the ladder axiom if for every commutative diagram in $\mathcal{M}$ of the form

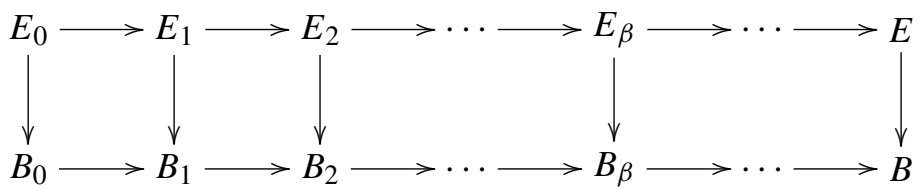


in which $B$ is the homotopy colimit of the bottom telescope of length $\lambda$ for some ordinal $\lambda$ and $E_{\beta}$ is the homotopy pull-back of $B_{\beta} \rightarrow B \leftarrow E$ for all $\beta<\lambda$, then $E$ is the homotopy colimit of the top line.

A model category (such as that of spaces) which satisfies both the cube axiom and the ladder axiom is easily seen to satisfy an even more general commutation axiom. Take any diagram $F: I \rightarrow \mathcal{M}$ indexed by a contractible category $I$ and any fibration $p: E \rightarrow \operatorname{hocolim}_{I} F$. Pull back the whole diagram along $p$ so as to obtain a diagram $G: I \rightarrow \mathcal{M}$ together with a natural transformation $F \rightarrow G$. Then $\operatorname{hocolim}_{I} G \simeq E$. This is precisely the cube axiom when $I$ is the push-out category $\bullet \leftarrow \bullet \rightarrow \bullet$ and the ladder axiom when $I$ is the telescope category $\bullet \rightarrow \bullet \rightarrow \bullet \rightarrow \cdots$.

We will actually not need the ladder axiom in its full generality, but are mainly interested in the commutation of telescopes with products. Sometimes it is easier to check such a property directly by using detectors, as defined in Definition 1.1.

Lemma 2.6. Let $\mathcal{M}$ be a model category either having a set of detectors $\mathcal{G}$ or satisfying the ladder axiom. Let $A$ be the homotopy colimit in $\mathcal{M}$ of a (possibly transfinite) telescope $A_{0} \rightarrow A_{1} \rightarrow A_{2} \rightarrow \cdots \rightarrow A_{\beta} \rightarrow \cdots$. Then, for any object $X \in \mathcal{M}$, $X \times A$ is the homotopy colimit of the diagram $X \times A_{0} \rightarrow X \times A_{1} \rightarrow X \times A_{2} \rightarrow$ $\cdots \rightarrow X \times A_{\beta} \rightarrow \cdots$.

Proof. When a set of detectors is available it suffices to compute the sets of homotopy classes out of the detectors $G \in G$ and check that

$$
[G, X \times A] \cong\left[G, \operatorname{hocolim}\left(X \times A_{0} \rightarrow X \times A_{1} \rightarrow X \times A_{2} \rightarrow \cdots\right)\right] .
$$

This is straightforward since the detectors are finite objects. In the case of a transfinite telescope of length $\lambda$ one should replace the object $X \times A_{\gamma}$ by the weakly equivalent one hocolim ${ }_{\alpha<\gamma} X \times A_{\alpha}$ for every limit ordinal $\gamma$ so as to get a true telescope by taking the product with $X$.

If the ladder axiom holds, it is simply the particular case when the fibration one pulls back is the trivial one $X \times A \rightarrow A$.

\section{The join}

We check here that all the classical facts about the join of spaces actually hold in any model category and introduce the join axiom. Most proofs here are not new, but probably folklore. Recall that the join $A * B$ of two objects $A, B \in \mathcal{M}$ is the homotopy push-out of $A \stackrel{p_{1}}{\longleftarrow} A \times B \stackrel{p_{2}}{\longrightarrow} B$. First notice that the induced maps $A \rightarrow A * B$ and $B \rightarrow A * B$ are trivial. Indeed the map $A \rightarrow A * B$ can be seen as 
the composite $A \stackrel{i_{1}}{\longrightarrow} A \times B \stackrel{p_{1}}{\longrightarrow} A \rightarrow A * B$ which by definition coincides with the obviously trivial map $A \stackrel{i_{1}}{\longrightarrow} A \times B \stackrel{p_{2}}{\longrightarrow} B \rightarrow A * B$.

Lemma 3.1. For any objects $A, B$ in a pointed model category $\mathcal{M}$, we have a weak equivalence $A * B \simeq \Sigma(A \wedge B)$.

Proof. We use a "classical" Fubini argument (homotopy colimits commute with themselves, cf. for example [4, Theorem 24.9]). Consider the commutative diagram

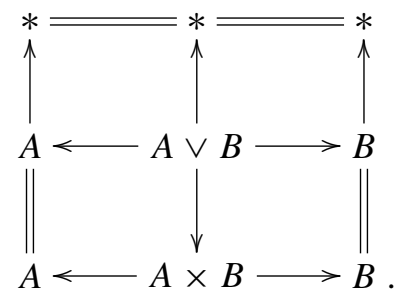

Its homotopy colimit can be computed in two different ways. By taking first vertical homotopy push-outs and next the resulting horizontal homotopy push-out one gets $\Sigma(A \wedge B)$. By taking first horizontal homotopy push-outs one gets $A * B$.

Lemma 3.2. For any objects $A, B$ in a pointed model category $\mathcal{M}$, we have a weak equivalence $\Sigma A \wedge B \simeq \Sigma(A \wedge B)$.

Proof. Apply again the Fubini commutation rule to the following diagram:

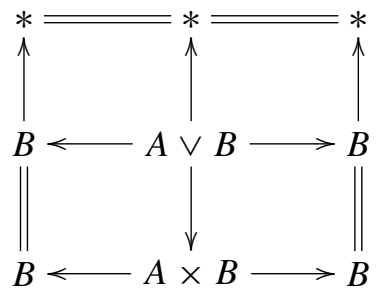

where one uses Lemma 2.4 to identify the push-out of the bottom line.

For a fibration $F \rightarrow E \rightarrow B$, the holonomy action is the map $m: \Omega B \times F \rightarrow F$ induced on the pull-backs by the natural transformation from $\Omega B \rightarrow * \leftarrow F$ to $P B \rightarrow B \leftarrow E$.

Corollary 3.3. For any fibration $F \rightarrow E \rightarrow B$ in a model category $\mathcal{M}$, the homotopy push-out of $\Omega B \leftarrow \Omega B \times F \stackrel{m}{\longrightarrow} F$ is weakly equivalent to $\Omega B * F$. 
Proof. Copy the proof of the preceding lemma to compare this homotopy push-out with $\Sigma(\Omega B \wedge F)$.

The next result, which is a particular case of Proposition 2.3, is a theorem originally due to Ganea (see [10]).

Proposition 3.4. Let $\mathcal{M}$ be a model category in which the cube axiom holds. Consider a fibration $F \rightarrow E \rightarrow B$, and the homotopy cofiber $E^{\prime}=\operatorname{Cof}(F \rightarrow E)$. The homotopy fiber $\mathrm{Fib}\left(E^{\prime} \rightarrow B\right)$ is then weakly equivalent to the join $F * \Omega B$.

Proof. The homotopy push-out of fibers one has to compute using Puppe's Proposition 2.3 is precisely that of Corollary 3.3.

Proposition 3.5. Let $\mathcal{M}$ be a model category in which the cube axiom holds. Assume also either that it has a set of detectors, or that it satisfies the ladder axiom. Then the join commutes with coproducts, push-outs, and telescopes.

Proof. The case of push-outs and telescopes follows from Lemmas 2.4 and 2.6. In the case of finite coproducts, notice that $X *(A \amalg B) \simeq(X * A) \bigsqcup(X * B)$ and for infinite ones, use the appropriate telescope.

Definition 3.6. A cellular model category $\mathcal{M}$ satisfies the join axiom for the nullification functor $P_{A}$ if the join of $A$ with the cofiber of any generating cofibration is $A$-acyclic.

Example 3.7. Any stable model category satisfies trivially the join axiom, as pushouts coincide with pull-backs. In such a category the join is always trivial. The category of groups satisfies the join axiom for a similar reason (but we saw in Example 2.2 that the cube axiom does not hold). The category of pointed spaces satisfies as well the join axiom. Here the cofibers of the generating cofibrations are spheres $S^{n}$ and the join $S^{n} * A \simeq \Sigma^{n+1} A$ is obviously $A$-acyclic.

Proposition 3.8. Let $\mathcal{M}$ be a cellular model category in which the join axiom and the cube axiom hold. Assume also that $\mathcal{M}$ either has a set of detectors, or satisfies the ladder axiom. Then $\Sigma^{i} A * Z$ is $A$-acyclic for any $i \geq 0$ and any object $Z$.

Proof. Any object in $\mathcal{M}$ has a cofibrant approximation which can be constructed from * as a telescope $C_{0} \rightarrow C_{1} \rightarrow C_{2} \rightarrow \cdots \rightarrow C_{\beta} \rightarrow \cdots$ of push-outs along generating cofibrations. Since $C_{0}$ and $\operatorname{Cof}\left(C_{0} \rightarrow C_{1}\right)$ are cofibers of generating cofibrations, both $A * C_{0}$ and $A * \operatorname{Cof}\left(C_{0} \rightarrow C_{1}\right)$ are $A$-acyclic. The join with $A$ of a cofibration is again a cofibration by Proposition 3.5, which proves next that $A * C_{1}$ is $A$-acyclic as well. A transfinite induction argument shows that $A * Z$ is $A$-acyclic since $A * C_{\gamma}$ is 
weakly equivalent to hocolim $\operatorname{lo\gamma }_{\alpha<\gamma}\left(A * C_{\alpha}\right)$ for any limit ordinal $\gamma$ by Proposition 3.5 again. We conclude now by Lemma 3.2 since $\Sigma^{i} A * Z \simeq \Sigma^{i}(A * Z)$ is $P_{A}$-acyclic for any $i \geq 0$.

Theorem 3.9. Let $\mathcal{M}$ be a cellular model category in which the join axiom and the cube axiom hold. Assume also that $\mathcal{M}$ either has a set of detectors, or satisfies the ladder axiom. Then $X * Z$ is $A$-acyclic for any object $Z$ and any $A$-acyclic object $X$.

Proof. Since $X$ is $A$-acyclic, one obtains a contractible object by iteratively attaching cones over suspensions of $A$ to $X$. Let us consider the first step $X_{1}=$ $\operatorname{Cof}\left(\bigvee_{i \geq 0} \bigvee \Sigma^{i} A \rightarrow X\right)$. By Proposition $3.5 X_{1} * Z$ is weakly equivalent to $\operatorname{Cof}\left(\bigvee_{i \geq 0} \bigvee \Sigma^{i} A * Z \rightarrow X * Z\right)$. As $\Sigma^{i} A * Z$ is $A$-acyclic by the above proposition, the map $X * Z \rightarrow X_{1} * Z$ is a $P_{A}$-equivalence. Iterating the process yields the desired $P_{A}$-equivalence from $X * Z$ to a contractible object. Notice that for any limit ordinal $\gamma$ the morphism $X * Z \rightarrow \operatorname{hocolim}_{\alpha<\gamma}\left(X_{\alpha} * Z\right)$ is a $P_{A}$-equivalence.

Remark 3.10. Given a family $F$ of generating cofibrations, we say $\mathcal{M}$ satisfies the restricted join axiom if the join of $A$ with the cofiber of any cofibration in $F$ is $A$ acyclic. One refines then the above proposition to cellular model categories in which the restricted join axiom holds. Here $\Sigma^{i} A * Z$ is $A$-acyclic for any $i \geq 0$ and any $F$-cellular object $Z$, i.e. any object weakly equivalent to one which can be built from $*$ by performing push-outs along cofibrations in $F$.

\section{Fiberwise localization}

The elegant construction proposed by Dror Farjoun in [8, 1.F.2] cannot work in an arbitrary pointed model category. The idea would be to decompose a fibration $E \rightarrow B$ as an unpointed homotopy colimit over the "simplices" of the base (simplices should be contractible objects). In particular $B$ would be expressed as a certain homotopy colimit of contractible objects. But if there is no underlying unpointed category available (think of differential graded Lie algebras for example), any (pointed) homotopy colimit of a diagram made of contractible objects will be contractible. Therefore there is a need for an alternative construction and we explain now how to adapt the fiberwise construction [8, 1.F.7] in a model category. The following lemma is the step we will iterate on and on so as to construct the space $\bar{E}$ (in Theorem 4.3). 
Proposition 4.1. Let $\mathcal{M}$ be a left proper and pointed model category in which the cube axiom holds. Consider a commutative diagram

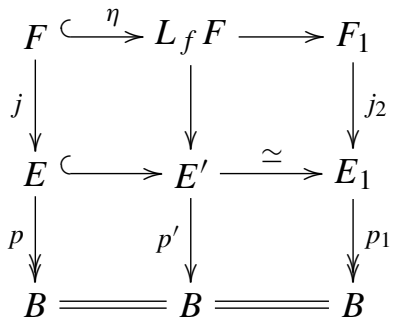

where the left column is a fibration sequence, the upper left square is a homotopy push-out square, $p^{\prime}: E^{\prime} \rightarrow B$ is the unique map extending $p$ such that the composite $L_{f} F \rightarrow E^{\prime} \rightarrow B$ is trivial, $p_{1}$ is a fibration, and $F_{1}$ is the homotopy fiber of $p_{1}$. Assume that the canonical map $F \times \Omega B \rightarrow L_{f} F \times \Omega B$ is an $f$-local equivalence. Then the composites $E \rightarrow E^{\prime} \rightarrow E_{1}$ and $F \rightarrow L_{f} F \rightarrow F_{1}$ are both $f$-local equivalences.

Proof. We can assume that the map $\eta: F \hookrightarrow L_{f} F$ is a cofibration as indicated in the diagram, so that $E^{\prime}$ is obtained as a push-out, not only a homotopy push-out. Since $\eta$ is an $f$-local equivalence, so is its push-out along $j$ by left properness (see [11, Proposition 3.5.4]). To prove that $F \rightarrow F_{1}$ is an $f$-local equivalence, it suffices to analyze the map $L_{f} F \rightarrow F_{1}$. We use Puppe's Proposition 2.3 to compute $F_{1}$ as homotopy push-out of the homotopy fibers of $L_{f} F \leftarrow F \rightarrow E$ over the fixed base $B$. This yields the diagram $L_{f} F \times \Omega B \leftarrow F \times \Omega B \rightarrow F$ whose homotopy push-out is $F_{1}$. By assumption the morphism $F \times \Omega B \rightarrow L_{f} F \times \Omega B$ is an $f$-local equivalence, thus so is $F \rightarrow F_{1}$.

Example 4.2. The category of pointed spaces is the prototype of a cellular model category in which the cube axiom holds. However, as shown by Hirschhorn in [11, Proposition 6.1.4], fiberwise localization does not always exist here! The above construction fails when the base is not 1-connected, because $\Omega B$ is not connected. When for example $B=S^{1}$ and $L_{f} F \simeq *$, it is not true that the map $F \times \mathbb{Z} \rightarrow \mathbb{Z}$ is an $f$-local equivalence, since $L_{f}(F \times \mathbb{Z})=F \times(\mathbb{Z} \backslash 0) \bigsqcup *$.

Theorem 4.3. Let $\mathcal{M}$ be a model category which is pointed, left proper, cellular and in which the cube axiom holds. Assume also that $\mathcal{M}$ either has a set of detectors, or satisfies the ladder axiom. Let $L_{f}: \mathcal{M} \rightarrow \mathcal{M}$ be a localization functor, $F \rightarrow E \rightarrow B$ be a fibration, and assume that $L_{f}(\Omega B \times X) \simeq L_{f} \Omega B \times L_{f} X$ for any $X \in \mathcal{M}$. Then 
there exists a fiberwise localization, i.e. a natural transformation to a new fibration

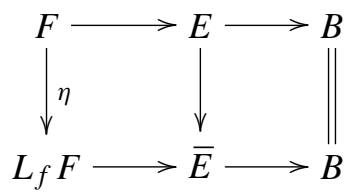

where $E \rightarrow \bar{E}$ is an $f$-local equivalence.

Proof. We construct first by the method provided in Lemma 4.1 a natural transformation to the fibration $F_{1} \rightarrow E_{1} \rightarrow B$. We iterate then this construction and define $\bar{E}=\operatorname{hocolim}\left(E \rightarrow E_{1} \rightarrow E_{2} \rightarrow \cdots\right), F_{\infty}=\operatorname{hocolim}\left(F \rightarrow F_{1} \rightarrow F_{2} \rightarrow \cdots\right)$, and $\bar{F}$ to be the homotopy fiber of $\bar{E} \rightarrow B$. If $\mathcal{M}$ satisfies the ladder axiom $F_{\infty}$ is weakly equivalent to $\bar{F}$ because the $F_{n}$ 's coincide with the homotopy pull-backs of $E_{n} \rightarrow \bar{E} \leftarrow \bar{F}$. If $\mathcal{M}$ has a set of detectors it is easy to see that the morphism $F_{\infty} \rightarrow \bar{F}$ induces isomorphisms $\left[G, F_{\infty}\right] \cong[G, \bar{F}]$ for any detector $G$.

We get thus a fibration $F_{\infty} \rightarrow \bar{E} \rightarrow B$. As all maps in the telescopes are $f$-local equivalences by the above lemma, hence so are $E \rightarrow \bar{E}$ and $F \rightarrow F_{\infty}$. Moreover any map $F_{n} \rightarrow F_{n+1}$ factorizes as $F_{n} \rightarrow L_{f} F_{n} \simeq L_{f} F \rightarrow F_{n+1}$ so that $F_{\infty} \simeq L_{f} F$. We obtain thus the desired fibration $L_{f} F \rightarrow \bar{E} \rightarrow B$.

Remark 4.4. The fiberwise localization we have constructed in Theorem 4.3 is obtained in a countable number of steps. This can be done because the ladder axiom or the detectors allow us to identify the homotopy fiber of $\bar{E} \rightarrow B$ with the homotopy colimit of the countable telescope of homotopy fibers $F_{n}$. In an arbitrary cellular model category this is not necessarily true and one would construct $\bar{E}$ by iterating the construction of Lemma 4.1 a transfinite number of times, up to the size of the cells of $\mathcal{M}$, see [11, Definition 12.3.3].

\section{Fiberwise nullification}

In general it is a very difficult problem to determine if a localization functor commutes with products. In the case of a nullification functor we will refine the analysis of the construction in Proposition 4.1 so as to use joins instead of products. Recall that when $f$ is a morphism of the form $A \rightarrow *, L_{f}$ is called nullification or periodization, and denoted by $P_{A}$.

The advantage of a nullification $X \rightarrow P_{A} X$ is that it can be constructed up to homotopy by imitating the topological construction 2.8 in [2]. One must iterate (possibly transfinitely, for a cardinal depending on the size of the cells of $\mathcal{M}$ and 
defined in [11, Definition 4.5.3]) the process of gluing $A$-cells, i.e. take the homotopy cofiber of a map $\Sigma^{i} A \rightarrow X$. We assume throughout this section that the model category $\mathcal{M}$ satisfies both the join axiom and the cube axiom.

Definition 5.1. An object $B$ is path connected if a morphism $E \rightarrow B$ is a weak equivalence if and only if its homotopy fiber is trivial.

Lemma 5.2. Let $\mathcal{M}$ be a model category which is pointed, left proper, cellular, in which the cube axiom and the join axiom hold. Let $F \rightarrow E \rightarrow B$ be a fibration with path connected base $B$. If $P_{A} F \simeq *$, then $P_{A} E \simeq P_{A} B$.

Proof. By Proposition 3.4 we see that the fiber $F_{1}$ of our iterative step of the fiberwise construction (Proposition 4.1) is $F * \Omega B$. This is an $A$-acyclic object by Theorem 3.9 and we noticed in Section 3 that the map $F \rightarrow F * \Omega B$ is trivial. Iterating the process as in Ganea's original construction, we see that the homotopy colimit $F_{\infty}$ of the successive homotopy fibers is contractible. Therefore the fiberwise nullification $\bar{E}$ is weakly equivalent to $B$. Moreover the map $E \rightarrow \bar{E}$ is a $P_{A}$-equivalence, since we kill $A$-acyclic fibers at each step.

Let us investigate more closely the map $F \rightarrow F_{1}$ in Proposition 4.1 by decomposing the map $F \rightarrow P_{A} F$ into several steps obtained by gluing $A$-cells.

Theorem 5.3. Let $\mathcal{M}$ be a model category which is pointed, left proper, cellular, in which the cube axiom and the join axiom hold. Let $P_{A}: \mathcal{M} \rightarrow \mathcal{M}$ be a nullification functor and $B$ an object such that $\Omega B$ is path connected. Assume also that $\mathcal{M}$ has a set of detectors, or satisfies the ladder axiom. Then there exists a fiberwise nullification, i.e. a construction which associates to any fibration $F \rightarrow E \rightarrow B$ another fibration together with a natural transformation

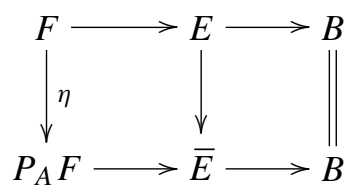

where $E \rightarrow \bar{E}$ is a $P_{A}$-equivalence.

Proof. Consider a cofibration of the form $\Sigma^{i} A \stackrel{f}{\longrightarrow} F \rightarrow C_{f}$. Let $E_{f}$ be the homotopy push-out of $C_{f} \leftarrow F \rightarrow E$ and compute as in Proposition 4.1 the homotopy fiber $F_{f}$ of $E_{f} \rightarrow B$. It is weakly equivalent to the homotopy push-out of $C_{f} \times \Omega B \leftarrow F \times \Omega B \rightarrow F$. Hence $F_{f}$ is also weakly equivalent to the homotopy push-out of $\Omega B \leftarrow \Sigma^{i} A \times \Omega B \rightarrow F$, using the definition of $C_{f}$. The first map is 
a $P_{A}$-equivalence because the projection $\Sigma^{i} A \times \Omega B \rightarrow \Omega B$ is one by Lemma 5.2. Thus so is $F \rightarrow F_{f}$ by left properness. The map $F \rightarrow F_{1}$ is obtained by repeating this process of gluing $A$-cells. It is therefore a telescope of $P_{A}$-equivalences, hence a $P_{A}$-equivalence. We can then conclude as in Theorem 4.3 that $F \rightarrow F_{\infty}$ is an $A$-nullification map.

As usually, let us denote by $\bar{P}_{A} X$ the homotopy fiber of the nullification map $\operatorname{Fib}\left(X \rightarrow P_{A} X\right)$. As in the case of spaces we get:

Corollary 5.4. Let $\mathcal{M}$ be a model category which is pointed, left proper, cellular, in which the cube axiom and the join axiom hold. Assume also that $\mathcal{M}$ has a set of detectors, or satisfies the ladder axiom. For any object $X$ in $\mathcal{M}$ such that $\Omega P_{A} X$ is path connected, we have $P_{A} \bar{P}_{A} X \simeq *$.

Proof. Apply the fiberwise localization to the fibration $\bar{P}_{A} X \rightarrow X \rightarrow P_{A} X$. This yields a fibration $P_{A} \bar{P}_{A} X \rightarrow \bar{X} \rightarrow P_{A} X$ in which the base and the fiber are $A$-local. Therefore $\bar{X}$ is $A$-local as well. But then $\bar{X} \simeq P_{A} X$ and so $P_{A} \bar{P}_{A} X$ is contractible.

We end this section with a characterization of the model categories which admit fiberwise nullifications. We assume for simplicity that all morphisms in the model category which have contractible fiber are weak equivalences. This assumption could be removed if one would work with fibrations over a base $B$ such that $\Omega B$ is path connected.

Theorem 5.5. Let $\mathcal{M}$ be a model category which is pointed, left proper, cellular and in which the cube axiom holds. Assume also that $\mathcal{M}$ has a set of detectors, or satisfies the ladder axiom, and that all objects are path connected. Then the following conditions are equivalent:

(i) The nullification functor $P_{A}$ admits a fiberwise version.

(ii) The nullification functor $P_{A}$ preserves finite products.

(iii) The canonical projection $X \times A \rightarrow X$ is a $P_{A}$-equivalence for any $X \in \mathcal{M}$.

(iv) The join axiom for $A$ is satisfied.

Proof. We prove first that (i) implies (ii). Apply the fiberwise nullification to the trivial fibration $X \rightarrow X \times Y \rightarrow Y$ to get a new fibration $P_{A} X \rightarrow E \rightarrow Y$. The inclusion of the fiber admits a retraction $E \rightarrow P_{A} X$, i.e. $E \simeq P_{A} X \times Y$. Applying this time the fiberwise nullification to $Y \rightarrow Y \times P_{A} X \rightarrow P_{A} X$, we see that the map $X \times Y \rightarrow P_{A} X \times P_{A} Y$ is a $P_{A}$-equivalence. As a product of local objects is local, this means precisely that $P_{A}(X \times Y) \simeq P_{A} X \times P_{A} Y$. 
Property (iii) is a particular case of (ii). We show now that (iii) implies (iv). If the canonical projection $X \times A \rightarrow X$ is a $P_{A}$-equivalence, the push-out of it along the other projection yields another $P_{A}$-equivalence, namely $A \rightarrow X * A$. Therefore the join $X * A$ is $P_{A}$-acyclic. Finally (iv) implies (i) as shown in Theorem 5.3.

The construction we propose for fiberwise nullification does not translate to the setting of general localization functors. We do not know if the cube and join axioms are sufficient conditions for the existence of fiberwise localizations.

\section{Algebras over an operad}

In this section we provide the motivating example for which this theory has been developed. For a fixed field $k$, we work with $\mathbb{Z}$-graded differential $k$-vector spaces $(k$-dgm) and consider the category $\mathcal{O}$-alg of algebras in $k$-dgm over a cofibrant operad $\mathcal{O}$ (the operads we are working with are zero in negative degrees). These are indeed pointed, right proper and cellular categories. Weak equivalences are quasiisomorphisms and fibrations are epimorphisms (complete references for the model category structures on operads and algebras over operads are [1] and [20]). Notice also that all objects are path connected and the free algebras on one generator $\mathcal{O}(x)$ form a set of detectors. For our purpose we also need to prove that this category is left proper. This proof can be generalized to a topological context or a simplicial one (see [19]).

Proposition 6.1. Let $R$ be a unitary commutative ring and consider the operads in the category of $\mathbb{Z}$-graded differential $R$-modules. Let $\mathcal{O}$ be a cofibrant operad. Then the category of $\mathcal{O}$-algebras is left proper.

Proof. As retracts of quasi-isomorphisms are quasi-isomorphisms in the category of $\mathcal{O}$-algebras and since this category is also cofibrantly generated, it suffices to prove that the push-out of a weak-equivalence along a generating cofibration is a weakequivalence. This is done in the case of cofibrant operads in a very general context by Spitzweck ([20, Theorem 4]).

We do not know if the join axiom holds in full generality for any object $A$. It does so however when $A$ is acyclic with respect to Quillen homology, which is the case we are most interested in, or when $A$ is a free algebra. We check that the cube axiom always holds, following the strategy of [6, Proposition A.15], which guarantees the existence of fiberwise versions of the plus-construction and Postnikov sections. In the case of $\mathbb{N}$-graded $\mathcal{O}$-algebras (the case $\mathcal{O}=\mathcal{A} s$ is treated by Doeraene) one has to restrict to a particular set of fibrations (the so-called $J$-maps), because they must 
be surjective in each degree in order to compute pull-backs. In the $\mathbb{Z}$-graded context all fibrations are epimorphisms, so that the cube axiom holds in full generality.

Theorem 6.2. Let $\mathcal{O}$ be a cofibrant operad. Then the cube axiom holds in the category $\mathcal{O}$-alg.

Proof. Let us briefly recall the key steps in Doeraene's strategy. We consider a push-out square of $\mathcal{O}$-algebras (along a generic cofibration $B \hookrightarrow B \bigsqcup \mathcal{O}(V)$ ):

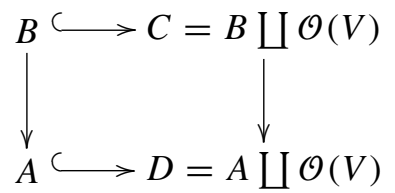

We need to compute the pull-back of this square along a fibration $p: E \rightarrow D$ (which is hence an epimorphism of chain complexes). We have thus the following isomorphism of chain complexes:

$$
E \cong A \coprod \mathcal{O}(V) \oplus \operatorname{ker}(p)
$$

The homotopy push-out $P$ of the successive pull-backs $A \times_{D} E, C \times_{D} E$, and $B \times{ }_{D} E$ must coincide with $E$. In order to prove this we factorize the morphism $B \times{ }_{D} E \rightarrow C \times{ }_{D} E$ as

$$
B \times_{D} E \hookrightarrow(B \oplus \operatorname{ker} p) \coprod \mathcal{O}(V \oplus W) \stackrel{\sim}{\rightarrow} C \times_{D} E
$$

Thus $P$ is identified with $(A \oplus \operatorname{ker}(p)) \bigsqcup \mathcal{O}(V \oplus W)$, which allows us to build finally a quasi-isomorphism to $E$.

We consider first the case of Postnikov sections $P_{\mathcal{O}(x)}$, where $x$ is a generator of arbitrary degree $n \in \mathbb{Z}$. Because $[\mathcal{O}(x), X] \cong \pi_{n} X$ for any $\mathcal{O}$-algebra $X$, the nullification functor $P_{\mathcal{O}(x)}$ is really a Postnikov section, i.e. $P_{\mathcal{O}(x)} X \simeq X[n-1]$. Let us also recall that $\pi_{n}(X \times Y) \cong \pi_{n} X \times \pi_{n} Y$.

Proposition 6.3. Let $\mathcal{O}$ be a cofibrant operad. The join axiom holds for any free $\mathcal{O}$-algebra $\mathcal{O}(x)$ on one generator of degree $n \in \mathbb{Z}$. Therefore fiberwise Postnikov sections exist in $\mathcal{O}$-alg.

Proof. By Theorem 5.5 we might as well check that the map $X \times \mathcal{O}(x) \rightarrow X$ is a $P_{\mathcal{O}(x)}$-equivalence for any $\mathcal{O}$-algebra $X$. Clearly the $n$-th Postnikov section of the product $X \times \mathcal{O}(x)$ is equivalent to $X[n-1]$ and we are done.

In the above proposition it was way easier to check commutation of Postnikov sections with products than to check directly the join axiom. In this case indeed the 
join axiom states that the join $\mathcal{O}(x) * \mathcal{O}(y)$ of two "spheres" is always $P_{\mathcal{O}(x)}$-acyclic, i.e. has its homotopy groups concentrated in degrees $\geq n$. In particular when $y$ is a generator of negative degree $m$, this tells us that the join of two spheres is far from being a sphere of degree $n+m+1$.

Our main application is nevertheless the existence of fiberwise plus-construction. In the world of non-negatively graded $\mathcal{O}$-algebras, the plus-construction is a nullification with respect to a universal acyclic algebra $\mathcal{U}$. We refer to [5] for an explicit construction and nice applications. Let us remain for a moment in the category of $\mathbb{Z}$-graded $\mathcal{O}$-algebras, in which we consider the $\mathcal{U}$-nullification functor $P u$.

Proposition 6.4. Let $\mathcal{O}$ be a cofibrant operad. The join axiom holds for any (-1)connected acyclic $\mathcal{O}$-algebra $A$. It holds in particular for the universal acyclic algebra $U$ constructed in [5], so that the fiberwise $U$-nullification exists.

Proof. The join $A * X$ is weakly equivalent to $\Sigma A \wedge X$ by Lemmas 3.1 and 3.2. Since $\Sigma A$ is 0 -connected and acyclic, it is trivial by the Hurewicz Theorem [5, Theorem 1.1]. Thus $A * X \simeq *$ is always $P_{A}$-acyclic.

Let us go back now to the category $\mathcal{O}$-alg $\geq 0$ of $\mathcal{O}$-algebras in the category of $\mathbb{N}$-graded differential $k$-vector spaces and consider the adjoint pair of functors

$$
F: \mathcal{O}-\mathbf{a l g}_{\geq 0} \leftrightarrows \mathcal{O}-\mathbf{a l g}: \operatorname{Tr}
$$

given by the forgetful functor $F$ and the truncation $\operatorname{Tr}$. The truncation is defined already at the level of chain complexes by $\operatorname{Tr}(A)_{n}=A_{n}$ for any positive integer $n$ and $\operatorname{Tr}(A)_{0}=\operatorname{ker}\left(d: A_{0} \rightarrow A_{-1}\right)$. It extends to the categories of algebras since the operad $\mathcal{O}$ is zero in negative degrees. As $F$ is a left adjoint, it preserves weak equivalences and cofibrations. In particular the cone on $U$ is the same in both categories and the functor $P_{u}$ coincides on non-negatively graded $\mathcal{O}$-algebras in both categories. To avoid confusion though, we will from now on write $B^{+}$for the $U$-nullification of an object $B$ in the category $\mathcal{O}-\mathbf{a l g}_{>0}$ and call it the plus-construction of $X$, following the notation and terminology used in [5].

Theorem 6.5. Let $\mathcal{O}$ be a cofibrant operad. Then the fiberwise plus-construction exists in $\mathcal{O}$-alg $\mathbf{a l}_{\geq 0}$ for fibrations $p: E \rightarrow B$ inducing a surjection on $\pi_{0}$.

Proof. Let $p: E \rightarrow B$ be a fibration in $\mathcal{O}$-alg $>0$ inducing a surjection on $\pi_{0}$. As the fiber of $p$ has the same homotopy type as the homotopy fiber of $F(p)$, we can use Proposition 6.4 to construct the fiberwise $\mathcal{U}$-nullification in $\mathcal{O}$-alg. This yields a fibration $\bar{E} \rightarrow B$ which fiber has the homotopy type of $P u F$. Applying truncation produces the desired fibration $\operatorname{Tr}(\bar{E}) \rightarrow B$ in $\mathcal{O}-\mathbf{a l g}_{\geq 0}$ whose fiber is $F^{+}$(notice that $\bar{E}$ and $\operatorname{Tr}(\bar{E})$ have the same homotopy type, since the non-trivial homotopy groups of $\bar{E}$ are concentrated in non-negative degrees). 
Remark 6.6. It is not necessary to use the sophisticated truncation techniques to get a fiberwise plus-construction. If the fibration $p$ one starts with induces a surjection on $\pi_{0}$, one can factorize it in $\mathcal{O}-\mathbf{a l g}_{\geq 0}$ as a trivial cofibration followed by a fibration which is an epimorphism in each degree. Thus one can work with degreewise epimorphisms, which are the fibrations in the $\mathbb{Z}$-graded world. Therefore the fiberwise $U$-nullification gives directly the fiberwise plus-construction (the total space $\bar{E}$ is non-negatively graded).

Our final result is a particular case of Corollary 5.4. A direct proof (without fiberwise techniques) seems out of reach.

Theorem 6.7. Let $\mathcal{O}$ be a cofibrant operad. For any $B$ in the category $\mathcal{O}-\mathbf{a l g}>0$, the homotopy fiber $A B=\operatorname{Fib}\left(B \rightarrow B^{+}\right)$is then acyclic with respect to Quillen homology.

Proof. As all objects are path-connected in $\mathcal{O}$-alg, Corollary 5.4 applies. To conclude notice that the plus-construction $B \rightarrow B^{+}$induces indeed a surjection on $\pi_{0}$, so that the result holds as well in $\mathcal{O}-\mathbf{a l g}_{\geq 0}$.

\section{References}

[1] C. Berger and I. Moerdijk, Axiomatic homotopy theory for operads. Comment. Math. Helv. 78 (2003), 805-831. Zbl 1041.18011 MR 2016697

[2] A. K. Bousfield, Localization and periodicity in unstable homotopy theory. J. Amer. Math. Soc. 7 (1994), 831-873. Zbl 0839.55008 MR 1257059

[3] C. Casacuberta and A. Descheemaeker, Relative group completions. J. Algebra 285 (2005), 451-469. Zbl 02157742 MR 2125447

[4] W. Chachólski and J. Scherer, Homotopy theory of diagrams. Mem. Amer. Math. Soc. 155 (2002), no. 736. Zbl 1006.18015 MR 1879153

[5] D. Chataur, J. L. Rodríguez, and J. Scherer, Realizing operadic plus-constructions as nullifications. $K$-Theory 33 (2004), 1-21.

[6] J.-P. Doeraene, L.S.-category in a model category. J. Pure Appl. Algebra 84 (1993), 215-261. Zbl 0777.55007 MR 1201256

[7] J.-P. Doeraene and D. Tanré, Axiome du cube et foncteurs de Quillen. Ann. Inst. Fourier (Grenoble) 45 (1995), 1061-1077. Zbl 0833.55005 MR 1359839

[8] E. Dror Farjoun, Cellular spaces, null spaces and homotopy localization. Lecture Notes in Math. 1622, Springer-Verlag, Berlin 1996. Zbl 0842.55001 MR 1392221

[9] W. Dwyer, H. Miller, and J. Neisendorfer, Fibrewise completion and unstable Adams spectral sequences. Israel J. Math. 66 (1989), 160-178. Zbl 0686.55014 MR 1017160

[10] T. Ganea, A generalization of the homology and homotopy suspension. Comment. Math. Helv. 39 (1965), 295-322. Zbl 0142.40702 MR 0179791 
[11] P. S. Hirschhorn, Model categories and their localizations. Math. Surveys Monographs 99, Amer. Math. Soc., Providence, RI, 2003. Zbl 1017.55001 MR 1944041

[12] M. Hovey, Model categories. Math. Surveys Monographs 63, Amer. Math. Soc., Providence, RI, 1999. Zbl 0909.55001 MR 1650134

[13] M. Mather, Pull-backs in homotopy theory. Canad. J. Math. 28 (1976), 225-263. Zbl 0351.55005 MR 0402694

[14] M. Mather and M. Walker, Commuting homotopy limits and colimits. Math. Z. 175 (1980), 77-80. Zbl 0447.55004 MR 0595633

[15] J. P. May, Fibrewise localization and completion. Trans. Amer. Math. Soc. 258 (1980), 127-146. Zbl 0429.55004 MR 0554323

[16] V. Puppe, A remark on "homotopy fibrations". Manuscripta Math. 12 (1974), 113-120. Zbl 0277.55015 MR 0365556

[17] D. Quillen, Homotopical algebra. Lecture Notes in Math. 43, Springer-Verlag, Berlin 1967. Zbl 0168.20903 MR 0223432

[18] -, Higher algebraic $K$-theory. I. In Algebraic $K$-theory, I: Higher $K$-theories (Proc. Conf., Battelle Memorial Inst., Seattle, Wash., 1972), Lecture Notes in Math. 341, SpringerVerlag, Berlin 1973, 85-147. Zbl 0292.18004 MR 0338129

[19] C. Rezk, Every homotopy theory of simplicial algebras admits a proper model. Topology Appl. 119 (2002), 65-94. Zbl 0994.18008 MR 1881711

[20] M. Spitzweck, Operads, algebras and modules in general model categories. Preprint 2002.

Received December 17, 2003; revised April 22, 2005

David Chataur, U.M.R. CNRS 8524, U.F.R. de Mathématiques, 59655 Villeneuve d'Ascq Cédex, France

E-mail: david.chataur@math.univ-lille1.fr

Jérôme Scherer, Departament de Matemàtiques, Universitat Autónoma de Barcelona, 08193 Bellaterra, Spain

E-mail: jscherer@mat.uab.es 